\title{
ANÁLISE FILOGENÉTICA DE QUESTIDAE E CLITELLATA: O PROBLEMA DA PARAFILIA DE "POLYCHAETA"
}

\author{
André R. S. Garraffoni ${ }^{1}$ \\ Dalton S. Amorim²
}

\begin{abstract}
PHYLOGENETIC ANALISYS OF THE QUESTIDAE AND CLITELLATA: THE "POLYCHAETA" PARAPHYLY PROBLEM. Hypotheses about the phylogenetic relationships within Questidae, and the relationships of this taxon with Clitellata using parsimony analysis of 35 external and internal morphological characters of 36 terminal taxons are presented. The results show the position of Questidae within Scolecida and Clitellata. Within the Polychaeta, Clitellata is the sister group of the Questidae. The results also indicate that the Scolecida, Microdrilli, Polychaeta, Oligochaeta and Tubificidae are paraphyletic. Clitellata, Megadrilli, Questidae, Hirudinae are monophyletic.
\end{abstract}

KEYWORDS. Polychaeta, Questidae, Clitellata, Cladistics.

\section{INTRODUÇ̃̃O}

A família Questidae foi erigida para único gênero Questa, estabelecido por HARTMAN (1966) com a espécie-tipo Q. caudicirra Hartman, 1966, descrita para o sudoeste da Califórnia. Hobson (1970) propôs mais um gênero, espécie-tipo Novaquesta trifucata, procedente da região da Nova Inglaterra. Westheide (1981) e JAMIESON \& WebB (1984) descreveram respectivamente Q. media (Ilhas Galápagos) e Q. ersei (Grande Barreira de Corais, na Austrália). Giere \& Erséus (1998) sinonimizaram Novaquesta com Questa e ainda acrescentaram quatro espécies: Questa bicirrata (ilhas Mactan, Filipinas), $Q$. mediterranea (Isola di Ponza, costa oeste da Itália), Q. riseri (Ilhas Bermudas) e $Q$. paucibranchiata (Baia de Carrie Bow, em Belize). Estudos sobre a morfologia, fisiologia e ecologia dos questídeos foram realizados por Pettibone (1982), Fauchald \& Rouse (1997), Glasby (2000) e Rouse \& Pleijel (2001).

A característica morfológica mais peculiar desse grupo de poliquetas é o dimorfismo sexual, com a fêmea apresentando na região anterior um clitelo destinado às funções reprodutoras. Os animais são dióicos, sendo as estruturas reprodutoras confinadas a poucos segmentos (Somaschini \& Gravina, 1993; Delgado \& NuÑEz, 1994; GIERE \& ERSÉUS, 1998). A presença de uma estrutura semelhante a um clitelo em Questidae

1. Centro de Estudos do Mar, Universidade Federal do Paraná, Av. Beira Mar s/n, 83255-000, Caixa Postal 50002, Pontal do Sul, PR, Brasil. (agarraffoni@lycos.com)

2. Faculdade de Filosofia Ciências e Letras de Ribeirão Preto, Universidade de São Paulo, Av. Bandeirantes 3900, 14090-901 Ribeirão Preto, SP, Brasil. (dsamorim@usp.br) 
levantou a suspeita de uma eventual relação com Clitellata (GIERE \& RISE, 1981; JAMIESON, 1983a, b; JAMIESON \& WeBB, 1984; Giere \& ERSÉUs, 1998). Esses animais já foram chamados de "poliquetas oligoquetóides" por Giere \& Rise (1981), ainda que eles mesmos e virtualmente todos os autores posteriores tenham rejeitado a hipótese de relação direta entre os questídeos e os Clitellata.

Os objetivos são: verificar a hipótese de monofilia de Questidae e a hipótese de monofilia do táxon composto por Questidae + Clitellata; tentar determinar a posição destes táxons entre os Annelida.

\section{MATERIAL E MÉTODOS}

A fonte de dados empíricos utilizados para a organização e tratamento dos dados coletados foi a literatura primária pertinente às famílias de Polychaeta e Clitellata. Os caracteres foram codificados como estados múltiplos, tratados como ordenados (WILKInson, 1992, 1995) e não-ordenados (tab. I) e com pesos iguais. Posteriormente, foi realizada a pesagem sucessiva desenvolvida por FARRIS (1969), a qual permite que cada caráter seja pesado de acordo com um índice obtido através de uma primeira análise. Foi utilizado o índice de retenção reescalonado (FARRIS, 1989). Caracteres ausentes, incertos ou inaplicáveis foram codificados como "?". As matrizes de dados foram construídas utilizando o programa MacClade 3.01: analysis of phylogenetic and character evolution W. P. Maddison \& D. R. Maddison, 1996 e a análise de parcimônia realizada pelo programa PAUP: Phylogenetic analysis using parsimony version 3.1, D. L. Swofford, 1993, através da busca heurística, adição randômica, com 100 réplicas. Os ramos com comprimento zero foram colapsados, com o comando MULPARS ativado e os caracteres foram otimizados sob o procedimento ACCTRAN. As árvores obtidas foram visualizadas e analisadas com ajuda do programa McClade 3.01. O consenso utilizado na análise foi o estrito (SoKAl \& RoHLF, 1981). Na polarização dos caracteres foi utilizado o método de comparação por grupo externo (NIXON \& CARPENTER, 1993), utilizando a família Spionidae para o enraizamento do cladograma. As famílias que, segundo Rouse \& FAuchaLD (1997), compõem o clado Scolecida (Paraonidae, Scalibregmatidae, Orbiniidae, Cossuridae, Opheliidae, Maldanidae, Questidae, Arenicolidae e Capitellidae) foram tomadas como grupo interno. Todas as espécies da família Questidae foram utilizadas, uma vez que um dos objetivos é verificar a monofilia do grupo.

\section{RESULTADOS E DISCUSSÃO}

Os caracteres utilizados foram tratados como ordenados exceto os de número 10 e 32 .

1. Números de cerdas: 0 , mais que duas por feixe; 1 , duas por feixe; 2 , ausente.

Cerdas localizadas lateralmente nos anelídeos, dispostas em dois feixes, um mais dorsal e outro mais ventral. O número de cerdas em cada feixe não é fixo e varia nos diversos grupos dentro de Annelida. Somente nas famílias Haplotaxidae, Lumbriculidae e em Achantobdellida o número de cerdas por feixe é fixo. No grupo (Branchiobdellida + Euhirudinae) as cerdas estão ausentes.

2. Clitelo: 0 , ausente; 1 , presente.

A cinta glandular dos questídeos foi considerada homóloga ao clitelo dos Clitellata.

3. Órgãos reprodutores: 0 , sem tagma definido; 1 , com tagma definido.

Órgãos reprodutores em tagmas definidos, encontrados nos Questidae e nos Clitellata.

4. Átrio: 0 , ausente; 1 , rudimentar; 2 , desenvolvido.

$\mathrm{O}$ átrio, pequena dilatação localizada na base do ducto masculino, é caracterizado como uma glândula celular. Ausente em todos os poliquetas, Haplotaxidae, Megadrilli, Enchytraidae, Capilloventridae, Randiellidae, mas pode estar presente e 
Tabela I. Matriz usada na análise cladística dos grupos Scolecida e Clitellata (0-3 = ordens das séries de transformação; ? = caráter ausente de informações; *, subfamília de animais de água salgada; **, subfamília de animais de água doce).

\begin{tabular}{llllllllllllllllllllllllllllllllllll}
\hline Taxa & 1 & 2 & 3 & 4 & 5 & 6 & 7 & 8 & 9 & 1 & 1 & 1 & 1 & 1 & 1 & 1 & 1 & 1 & 1 & 2 & 2 & 2 & 2 & 2 & 2 & 2 & 2 & 2 & 2 & 3 & 3 & 3 & 3 & 3 & 3 \\
& & & & & & & & & 0 & 1 & 2 & 3 & 4 & 5 & 6 & 7 & 8 & 9 & 0 & 1 & 2 & 3 & 4 & 5 & 6 & 7 & 8 & 9 & 0 & 1 & 2 & 3 & 4 & 5 \\
\hline
\end{tabular}

\begin{tabular}{|c|c|c|c|c|c|c|c|c|c|c|c|c|c|c|c|c|c|c|c|c|c|c|c|c|c|c|c|c|}
\hline Spionidae & 0 & & & 0 & & & & & 0 & ? ? & ? & ? & 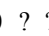 & & ? & 0 & & & & & & & & & & & & \\
\hline Scalibregmatidae & 0 & 0 & 0 & 0 & 0 & 0 & 0 & 0 & 0 & ? ? & $?$ & $?$ & $?$ & & & 0 & & 0 & 0 & & & 0 & & & & & & 1 \\
\hline Orbiniidae & 0 & 0 & 0 & 0 & 0 & 0 & 0 & 0 & 0 & ? ? & $?$ & $?$ & $?$ & & & 0 & & 0 & $\begin{array}{ll}0 & 1\end{array}$ & & & 0 & & & & & & \\
\hline Paraonidae & 0 & 0 & 0 & 0 & 0 & 0 & 0 & 0 & 0 & ? ? & ? & & $?$ & & & 0 & & 0 & 0 & & & & & & & & & \\
\hline Cossuridae & 0 & 0 & 0 & 0 & 0 & 0 & ( & 0 & 0 & ? ? & ? & $?$ & $?$ & & & 0 & & 0 & 0 & & & 0 & & & & & & \\
\hline Opheliidae & 0 & 0 & 0 & 0 & 0 & 0 & & 0 & 0 & ? ? & ? & ? & $?$ & & & 0 & & 0 & 0 & & & 0 & & & 0 & & & \\
\hline Maldanidae & 0 & 0 & 0 & 0 & 0 & 0 & 0 & 0 & 0 & ? ? & ? & & $?$ & & & 0 & & 0 & 0 & & & 0 & 0 & & 0 & & & \\
\hline Arenicolidae & 0 & 0 & 0 & 0 & 0 & 0 & 0 & 0 & 0 & ? ? & $?$ & & $?$ & & & 0 & & 0 & 0 & & 1 & 1 & & U & 1 & & & \\
\hline Capitellidae & 0 & 0 & 0 & 0 & 0 & 0 & 0 & 0 & 0 & ? ? & ? & ( & $?$ & & & 0 & & 0 & 0 & & 1 & 1 & & 0 & 1 & & & \\
\hline Q. caudicirra & 0 & 1 & 0 & & 0 & 0 & 0 & & 0 & & 0 & 1 & 0 & & & 1 & & 1 & 1 & & 0 & 0 & & & 1 & & & \\
\hline Q. media & 0 & 1 & 0 & & 0 & 0 & 0 & & 0 & $?$ & 0 & 1 & 0 & & ? & 1 & & & 0 & & 0 & 0 & & & 1 & & & \\
\hline Q. ersei & 0 & 1 & 0 & 0 & 0 & 0 & 0 & & 0 & ? ? & 0 & 1 & 0 & & & 1 & & 1 & 2 & & 0 & 0 & & & 1 & & & \\
\hline Q. trifurcata & $\begin{array}{ll}0 & 1\end{array}$ & 1 & 0 & 0 & 0 & 0 & 0 & 1 & 0 & ? ? & 0 & 1 & 0 & & ? & 1 & & 1 & 1 & & 0 & 0 & 1 & & 1 & & & \\
\hline Q. bicirrata & 0 & 1 & 0 & & 0 & 0 & 0 & & 0 & $?$ ? & 0 & $\left(\mathrm{C}-\mathrm{C}^{2}\right.$ & 0 & & ? & 1 & & 1 & 1 & & 0 & 0 & 1 & & 1 & & & \\
\hline Q. paucibranchiata & 0 & 1 & 0 & 0 & 0 & 0 & 0 & & 0 & ? ? & 0 & 1 & 0 & & ? & 1 & & 1 & 1 & & 0 & 0 & 1 & & 1 & & & \\
\hline diterran & 0 & 1 & 0 & 0 & 0 & 0 & 0 & 1 & 0 & ? ? & 0 & 1 & 0 & & ? & 1 & & 1 & 1 & & 0 & 0 & 1 & & 1 & & & \\
\hline O. 1 & 0 & 1 & 0 & 00 & 0 & 0 & 0 & 1 & 0 & ? ? & 0 & 1 & 0 & & $?$ & 1 & & 1 & 02 & & 0 & 0 & & & 1 & & & \\
\hline Haplotaxidae & 1 & 1 & 0 & 0 & 0 & 0 & 1 & 1 & 1 & 00 & 0 & 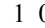 & 0 & & 1 & 1 & & 0 & 03 & & 0 & 1 & & 0 & 0 & & & \\
\hline Megadrilli & $?$ & 1 & 0 & 0 & 0 & 0 & 1 & 2 & 1 & $\begin{array}{ll}0 & 0\end{array}$ & 0 & 0 & 1 & & 0 & 1 & & 0 & 03 & & 0 & & & & 0 & & & \\
\hline aidae & 0 & 1 & 0 & 0 & 0 & 0 & 1 & 3 & 1 & 00 & 1 & 00 & 0 & & 1 & 2 & & 0 & 03 & & ) & & & & 0 & & & \\
\hline Capilloventridae & 0 & 1 & 0 & 0 & 0 & 0 & 1 & 3 & 1 & 20 & 1 & 00 & 0 & & 1 & 2 & & 0 & 3 & & 0 & 1 & & & 0 & & & \\
\hline Randiellidae & 0 & 1 & 0 & 0 & 0 & 0 & 1 & 3 & 1 & 20 & 1 & 00 & 0 & & 1 & 2 & U & 0 & 0 & & 0 & 1 & 0 & 0 & 0 & & & \\
\hline idae & 0 & 1 & 1 & 0 & 0 & 0 & 1 & 3 & 1 & 00 & 1 & 00 & 0 & & 1 & 1 & 1 & 0 & 03 & & 0 & 1 & 0 & 0 & 0 & & & \\
\hline idae & 0 & 1 & 2 & 0 & 0 & 0 & 1 & 3 & 1 & $\begin{array}{ll}0 & 0\end{array}$ & 1 & 01 & 0 & ) & 1 & 2 & 0 & 0 & 03 & & 0 & 1 & 0 & 0 & 0 & & & \\
\hline lidae & 1 & 1 & 2 & 1 & 0 & 1 & 1 & 3 & 1 & 00 & 1 & 01 & 0 & ) & 1 & 1 & 0 & 0 & 03 & & 0 & 1 & 0 & 0 & 0 & & & \\
\hline bdellida & 1 & 1 & 2 & 1 & 1 & 1 & 1 & 3 & 1 & 01 & 1 & 01 & 0 & ) & 1 & 1 & 0 & 0 & 03 & & 0 & 1 & 0 & 0 & 0 & & & \\
\hline iobdellida & 2 & 1 & 2 & 1 & 1 & 1 & 1 & 3 & 1 & 00 & 1 & 01 & 0 & & 1 & 1 & 0 & 0 & 0 & & 0 & 1 & 0 & 0 & 0 & & & \\
\hline Euhi & 2 & 1 & 2 & 1 & 2 & 0 & 1 & 3 & 1 & 00 & 1 & 00 & 0 & & 1 & 1 & 0 & 0 & 0 & & 0 & 1 & 0 & 0 & 0 & & & \\
\hline Phall & 0 & 1 & 2 & 1 & 0 & 0 & 1 & 3 & 1 & 10 & 1 & 0 & 0 & & 1 & 1 & 0 & 0 & 0 & & 0 & 1 & 0 & 0 & 0 & & & \\
\hline Ryancodriilidae* & 0 & 1 & 2 & 1 & 0 & 0 & 1 & 3 & & 10 & & 0 & 0 & & & 1 & & 0 & 0 & & 0 & 1 & 0 & & 0 & & & \\
\hline Ryancodriilidae** & 0 & 1 & 2 & & 1 & 0 & 1 & 3 & & 1 & & 0 & 0 & & & 2 & & 0 & & & & & & & 0 & & & \\
\hline Naididae & 0 & 1 & 2 & & 1 & 0 & 1 & 3 & & 1 & & 0 & 0 & & & 1 & & 0 & 0 & & & & & & 0 & & & \\
\hline cystidae & 0 & 1 & 2 & & 1 & 0 & 1 & 3 & & 00 & & 0 & 0 & & & 1 & & 0 & 0 & & & & & & 0 & & & \\
\hline drilini & 0 & & 2 & & & & 1 & 3 & & 0 & & & 0 & & & & & 0 & & & & & & & & & & \\
\hline Tubificinae & $\begin{array}{ll}0 & 1\end{array}$ & 1 & 2 & & 0 & 0 & 1 & 3 & & & & 00 & 0 & & & 1 & & 0 & $0 ?$ & & 0 & 1 & & 0 & 0 & & & \\
\hline Telmatodriilini & 0 & 1 & 2 & 11 & 10 & 0 & 1 & 3 & 1 & & & 00 & 0 & & & 1 & & 0 & & & 0 & 1 & 0 & 0 & 0 & & & \\
\hline
\end{tabular}

rudimentar em Pheodrilidae e presente e desenvolvido nos demais clitelatas (BRINKHURST, 1984a).

5. Glândula prostática: 0 , ausente; 1 , presente.

Glândula prostática, órgão de reprodução masculina, está relacionada ao átrio; é encontrada nos Lumbriculidae, Achantobdellida, Branchiobdellida, Euhirudinae, Phallodriilinae, Ryancodriilidae de água doce e salgada, Naididae, Poistocystidae, Limnodrilini, Tubificinae e Telmatodriilini(BRINKHURST, 1982, 1984a, 1994).

6. Sexo: 0, dióico; 1, hermafrodita.

Caráter utilizado para diferenciar os dois grupos existentes nos anelídeos: Polychaeta, com a condição dióica ou gonocorística e Clitellata, hermafroditas.

7. Ventosa: 0, ausente; 1, posterior; 2, posterior e anterior.

Ventosa encontrada somente nos táxons internos de Hirudinae (SidDAll \& BurReson, 1995). 

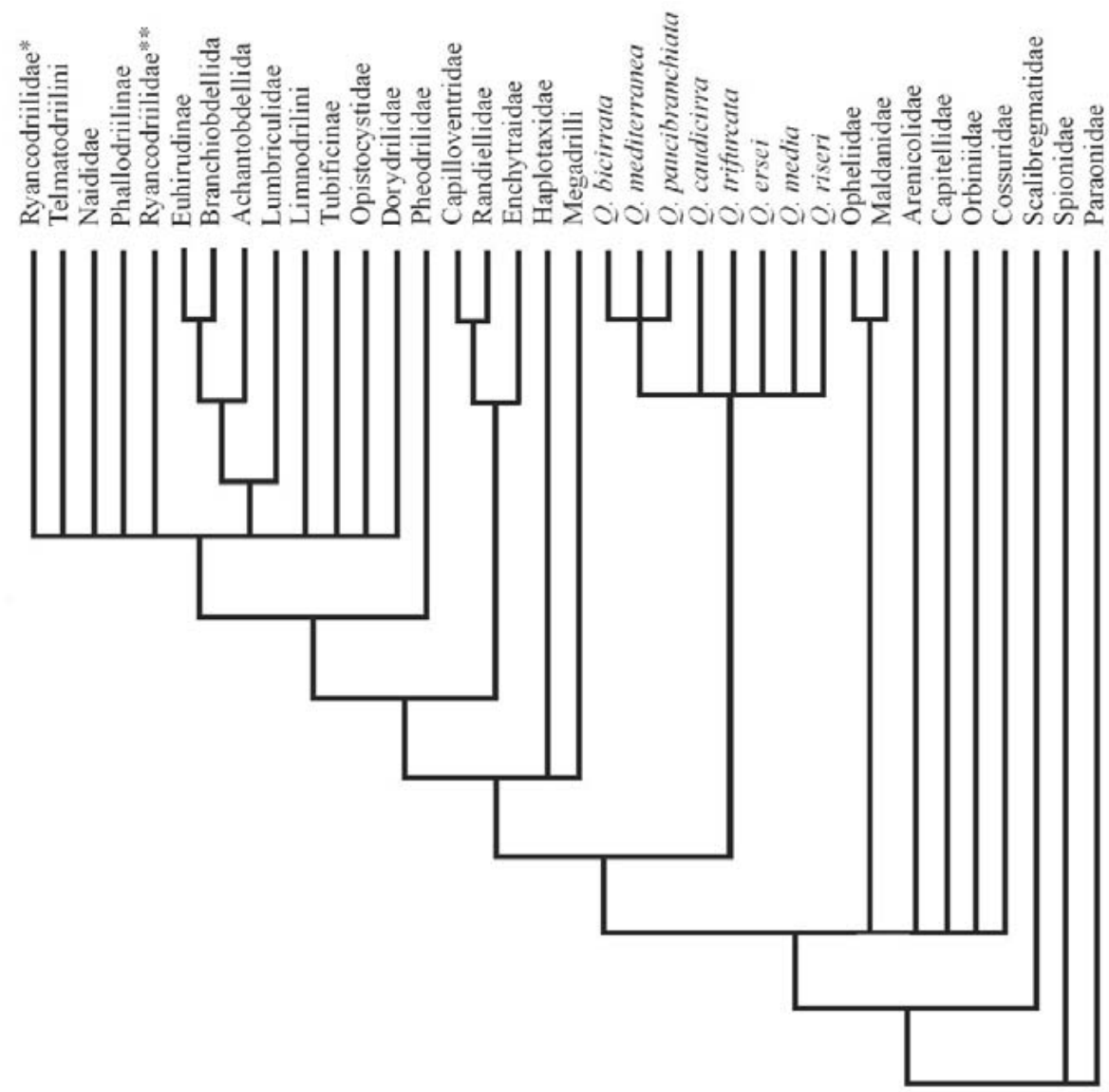

Fig. 1. Cladograma de consenso estrito obtido a partir da análise de caracteres codificados como estados múltiplos, apresentando as relações entre "Polychaeta" e os Clitellata (*, subfamília de animais de água salgada; **, subfamília de animais de água doce).

8. Segmentação: 0 , número variável de metâmeros; 1 , número fixo de metâmeros.

Nos Polychaeta e "Oligochaeta", os adultos, mesmo os da mesma espécie não apresentam um número fixo de metâmeros. Nos Hirudinae os diferentes grupos apresentam padrões quanto ao número de metâmeros: Acanthobdellida com 30 metâmeros, Branchiobdellida 15 e Euhirudinea 34.

9. Desenvolvimento: 0 , através de larva; 1 , direto.

Os poliquetos aqui estudados apresentam desenvolvimento por meio de larva planctônica. Nos Clitellata, os indivíduos imaturos são idênticos à forma adulta.

10.Ovário: 0 , ausente; 1 , dois pares; 2 , perda do par anterior; 3 , perda do par posterior.

Ausência de qualquer estrutura reprodutora, ativa em todo o período de vida, é 

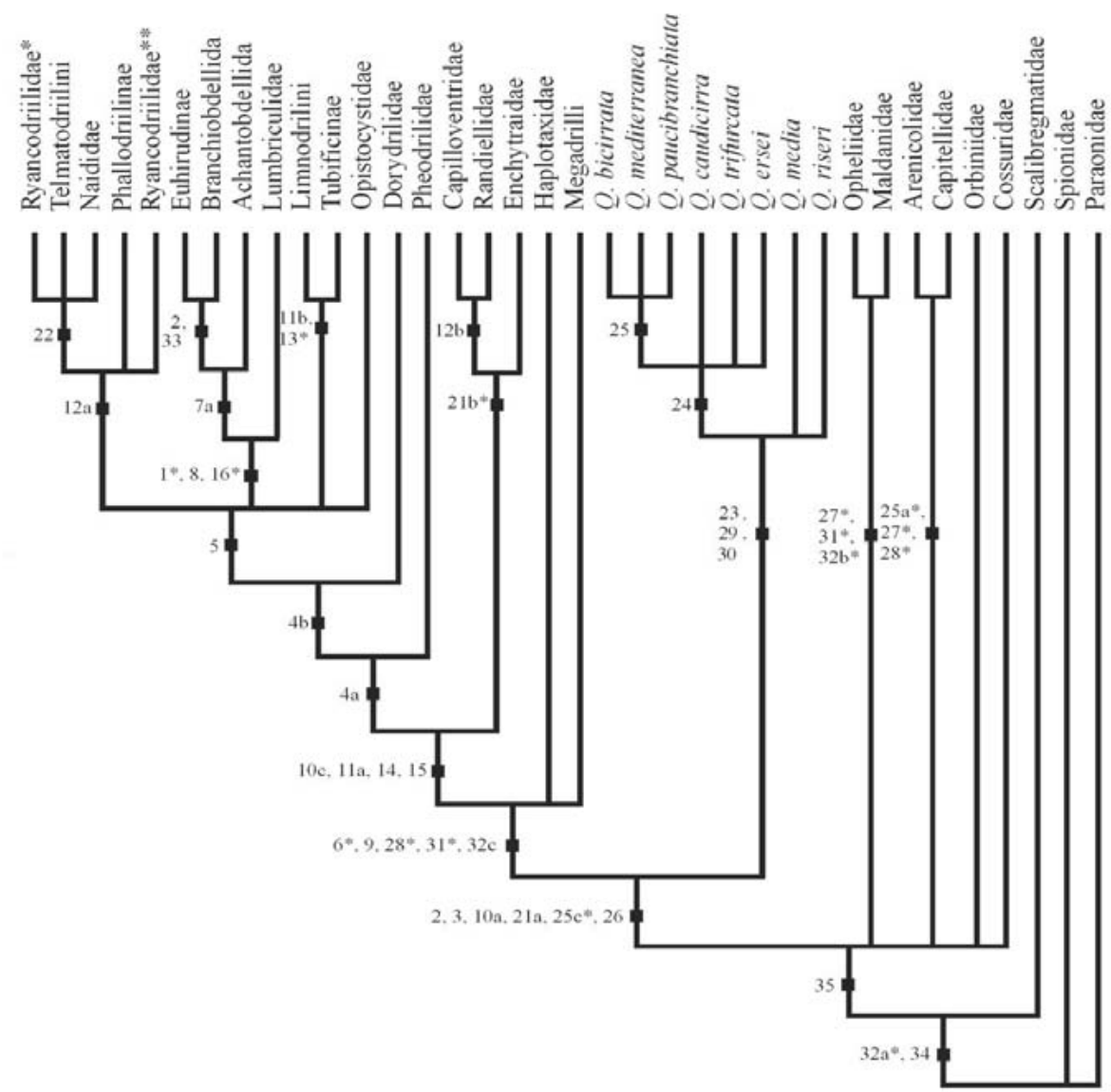

Fig. 2. Cladograma de ponderação sucessiva e consenso estrito obtido a partir da análise de caracteres codificados como estados múltiplos, apresentando as relações entre "Polychaeta" e os Clitellata, obtendo uma árvore c 439, ic 0.808 e ir $0.962 . *$, subfamília de animais de água salgada, **, subfamília de animais de água doce.

encontrada em quase todos os Polychaeta. Nas famílias Questidae e Haplotaxidae, o aparelho reprodutor feminino é completo e permanente com dois pares de ovários. Nos grupos Microdilli e Hirudinae, o aparelho reprodutor é completo, mas sem o segundo par de gônadas. Em Megadrilli, também é completo, contudo, não contém o primeiro par de ovários.

11. Histologia do átrio: 0 , ausente; 1 , homogêneo; 2 , heterogêneo.

Quase todos os clitelatas possuem a histologia do átrio homogênea. Contudo, Limnodrilini e os Tubificinae possuem o átrio heterogêneo (ERSÉUS, 1987).

12. Cerdas penianas: 0 , ausente; 1 , presente e simples; 2 , presente e ramificada. 
Especialização das cerdas relacionadas com um comportamento reprodutivo, presente de forma simples em Phallodriilinae, Ryancodriilidae de água doce e salgada e em Naididae. Capilloventridae e Randiellidae apresentam a forma ramificada (BRINKHURST \& NEMEC, 1987; BRINKHURST, 1994).

13. Papilas epidérmicas na parede do corpo: 0 , ausente; 1 , presente.

Presentes em Achantobdellida, Limnodrilini, Tubificinae e Telmatodriilini.

14. Gonoducto dos segmentos 13-14: 0, ausente; 1 , presente.

Presente em todos os clitelatas, com exceção de Haplotaxidae e Megadrilli.

15. Abertura do gonoducto no segmento 14: 0, ausente; 1, presente.

Presente apenas nos Questidae, Haplotaxidae e Megadrilli.

16. Deslocamento da abertura gonadal masculina para o metâmero onde está a gônada: 0 , ausente; 1 , presente.

Essa é a condição dita prosópora do aparelho reprodutor masculino, ou seja, tanto o testículo como o gonóporo e o ducto espermático estão localizados no mesmo metâmero. Os caracteres 17, 18, 19 e 20 são todos pequenas modificações no aparelho reprodutivo ocasionadas pelo aparecimento da condição opistópora e estão presentes apenas nos Megadrilli.

17. Fusão do gonoducto dos segmentos 10+11: 0 , ausente; 1, presente.

18. Deslocamento do gonóporo do segmento 11 para o 13: 0, ausente; 1, presente.

19. Gonoducto feminino nos segmentos 12-13: 0, presente; 1 , ausente.

20. Gonóporo masculino dos segmentos 11-12: 0, presente; 1, ausente.

21. Espermateca: 0, ausente; 1, localizada nos arredores do gonóporo; 2, localizada no segmento anterior ou em vários segmentos anteriores do gonóporo.

A presença da espermateca próximo ao gonóporo ocorre em Questidae e em quase todos os Clitellata, menos em Enchytraidae, Capilloventridae, Randiellidae e Dorydrilidae, que apresentam a abertura da espermateca no segmento anterior ao do gonóporo. A ausência de espermateca é verificada nos demais poliquetas estudados nesse trabalho.

22. Cerdas pectinadas: 0 , ausente; 1 , presente.

Encontradas em Pheodrilidae, Ryancodriilidae de água salgada, Naididae e Tubificinae (BRINKHURST \& NEMEC, 1987).

23. Posição dorsal ou dorso-lateral do gonóporo: 0 , ausente; 1 , presente.

Caráter presente apenas nos Questidae (Giere \& Rise, 1981; JAmieson \& WebB, 1984; Giere \& Erséus, 1998).

24. Anelamento secundário nos metâmeros: 0 , ausente; 1 , presente.

Presente em todas as espécies de Questidae, com exceção de Questa media e Q. riseri (Westheide, 1981; JAMieson \& WebB, 1984). 


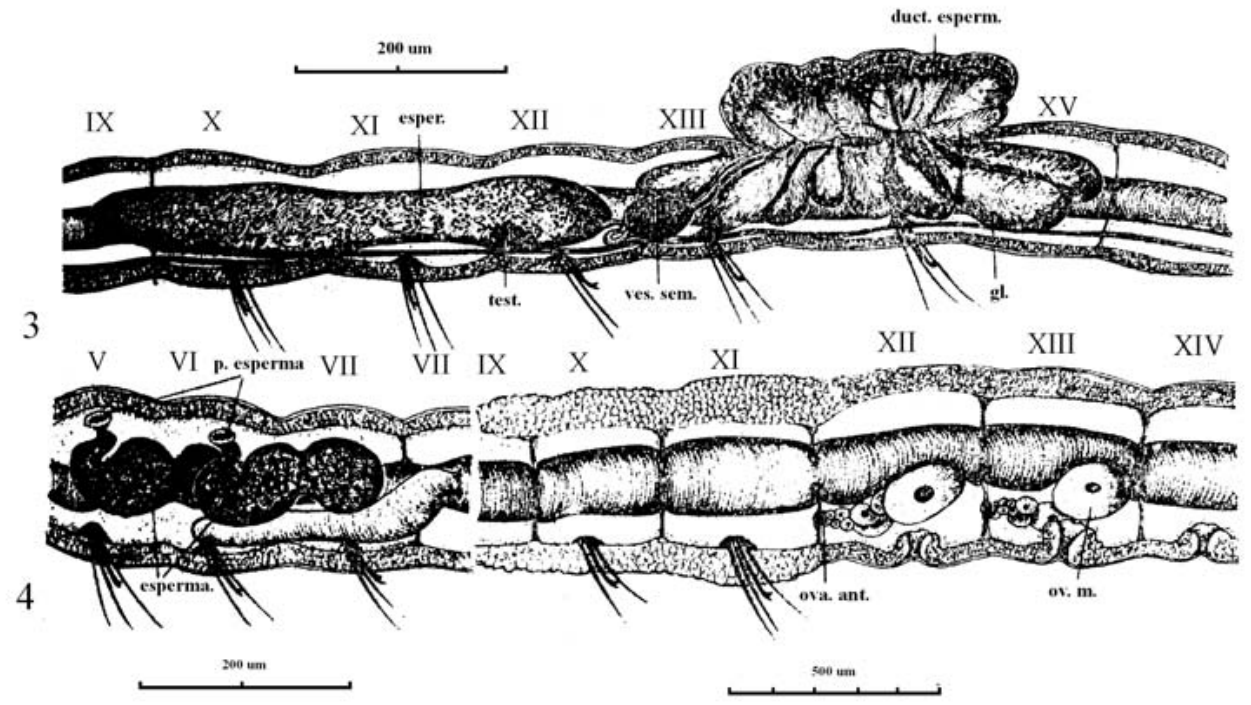

Figs. 3, 4. Diagrama esquemático dos órgãos genitais de Novaquesta trifurcata Hobson, 1970: 3 , masculino, 4, feminino (modificado de Giere \& Rise, 1981) (duct. esperm., ducto espermático; esperma., espermateca; esper., espermatozóide; p. esperma., poro da espermateca; test., testículo; ova. ant., ovário anterior; ves. sem., vesícula seminal; ov. m., óvulo maduro; gl., glândula mucosa).
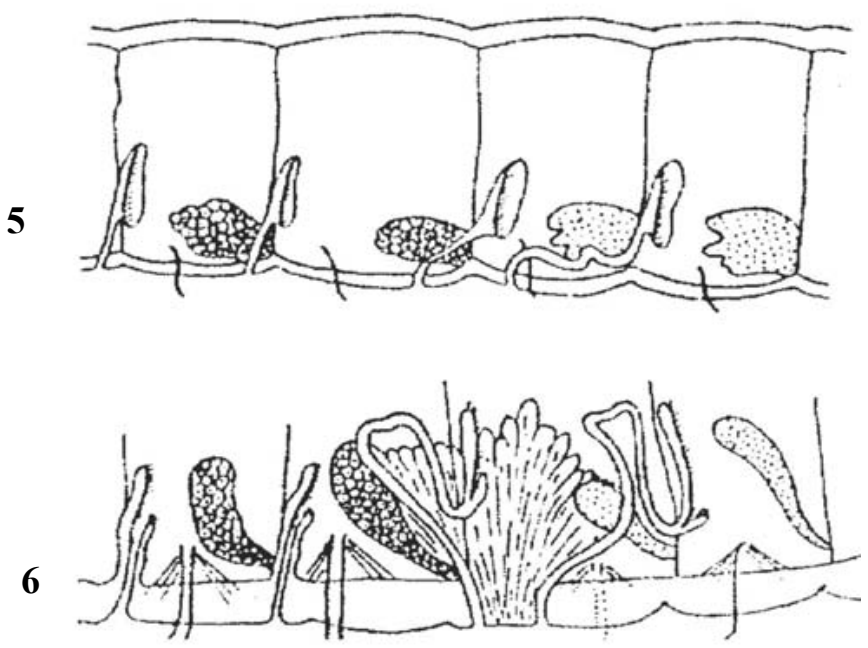

Figs. 5, 6. Vista lateral interna dos órgãos reprodutivos da família Haplotaxidae: 5, Haplotaxis heterogyne Benham, 1903; 6, H. africanus Michaelsen, 1905 (dois primeiros segmentos contêm os testículos, círculos escuros; dois primeiros segmentos posteriores contêm os ovários, círculos claros) (modificado de BRINKHURST \& JAMIESON, 1971). 


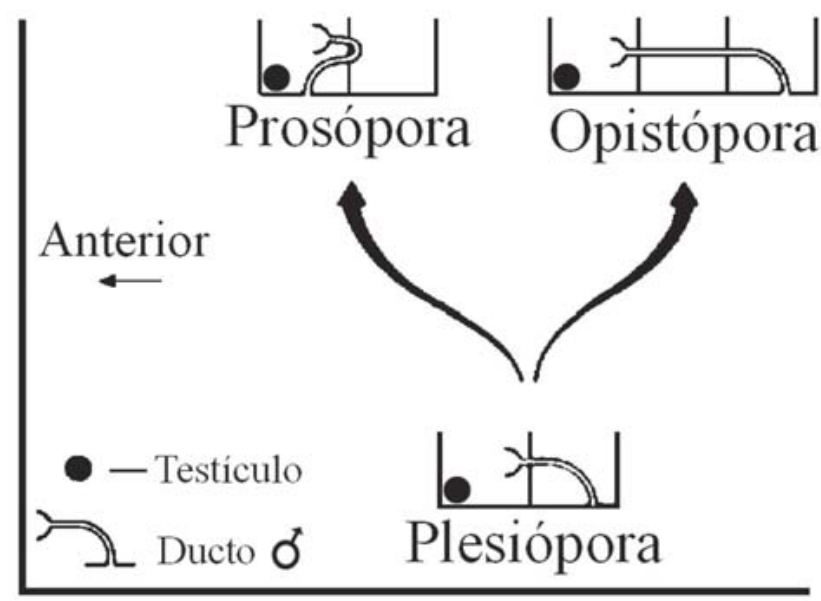

Fig. 7. Derivação dos ductos masculinos prosóporo e opistóporo da forma plesiópora ancestral (modificado de BRINKHURST, 1982).

25. Forma das cerdas: 0 , apenas o dente principal; 1 , bifurcada; 2 , trifurcada; 3 , ausente. Cerdas com apenas o dente principal, sem ramificações (Arenicolidae, Maldanidae, Capitellidae, Paraonidae e Orbiniidae), dente principal com ramificações apicais bífidas (Spionidae, Questa caudicirra, Q. media, Q. bicirrata, Q. paucibranchiata, Q. mediterranea), ramificações apicais trífidas (Q. trifurcata, $Q$. riseri e $Q$. ersei) ou ausente (Clitellata, Opheliidae, Scalibregmatidae e Cossuridae).

26. Orgãos nucais: 0 , ausente; 1 , presente.

Estruturas sensoriais, localizadas no prostômio, consideradas por FAUCHALD \& RousE (1997) como sinapomorfia dos "Polychaeta". Estudos recentes (PURSCHKE, 1997, 1999) provam que tal afirmação pode estar incorreta.

27. Membrana gular: 0 , presente; 1 , ausente.

Os septos encontrados no interior dos poliquetas são similares por toda extensão do corpo, ou sofrem pequenas modificações ao longo deste (FAuchald \& Rouse, 1997), encontrados em Capitellidae, Opheliidae, Arenicolidae e Maldanidae.

28. Cirro pigidial: 0 , presente com mais de dois pares; 1 , ausente.

Pequenas estruturas pós-segmentares localizadas no ânus; podem ser rugosas e variar tanto em número quanto em tamanho (FAuchald \& Rouse, 1997).

29. Par de grandes sacos espermáticos nos segmentos 12 e 13: 0, ausente; 1 , presente. Encontrado apenas na família Questidae (Giere \& Rise, 1981; JAmieson \& WebB, 1984).

30. Par de vesículas seminais no segmento 14 perto do septo do segmento 13 nos machos maduros, conectando os sacos espermáticos ao ducto: 0 , ausente; 1 , presente.

Encontrado apenas na família Questidae (GIERE \& RISE, 1981; JAMIESON \& WEBB, 1984). 


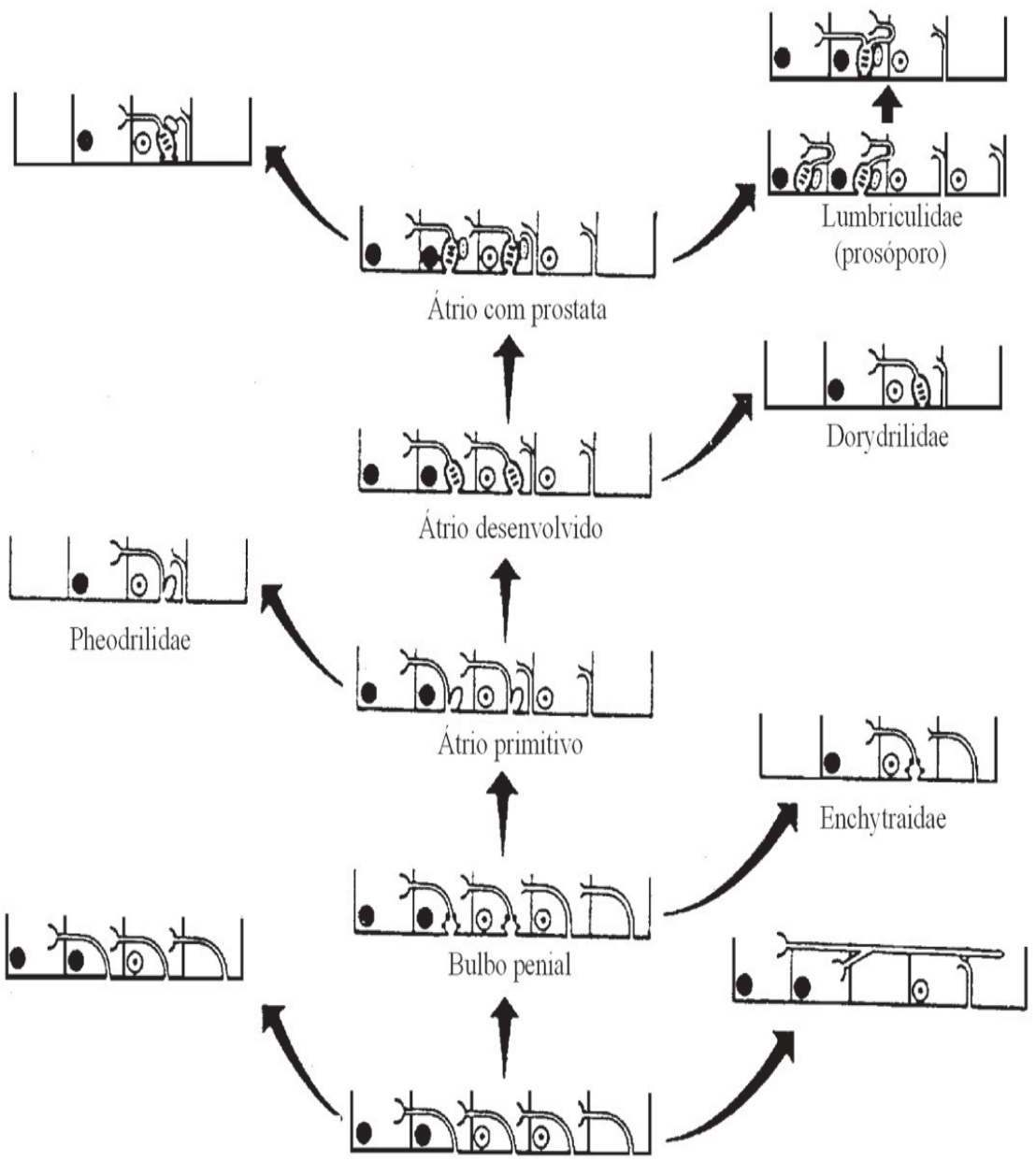

Forma basal de Haplotaxidae

(plesióporo)

gonoducto $\bigcirc$ testículo $\odot$ ovário

ל. gonoducto masculino com bulbo penial

\2 gonoducto masculino com átrio primitivo

gonoducto masculino com átrio desenvolvido

gonoducto masculino com glândula prostática

Fig. 8. Série de transformação do ducto masculino dos oligoquetos aquáticos (modificado de BRINKHURST, 1984b). 
31. Órgãos laterais: 0 , presente; 1 , ausente.

Órgãos laterais são sulcos ciliados internamente ou papilas ciliadas presentes entre o notopódio e o neuropódio (Fauchald \& Rouse, 1997). Presente em todos os poliquetas analisados, com exceção de Opheliidae e Maldanidae e em todos os Clitellata.

32. Parapódios: 0 , espionimorfa; 1 , reduzidos sem a formação de tori; 2 , reduzidos com a formação de tori; 3 , ausentes.

Parapódios desenvolvidos em Spionidae, ou reduzidos, limitados a pequenas expansões laterais (com ou sem cerdas encapuzadas), e ausentes nos Clitellata.

33. Pênis: 0 , ausente; 1 , presente.

Presente em Pheodrillidae, Branchiobdellida e Euhirudinae (BRINKHURST, 1992, 1994; BRINKHURST \& NEMEC, 1987).

34. Antena mediana: 0 , ausente; 1 , presente.

Apesar da incerteza de homologia entre a carúncula de Spionidae e a antena mediana dos poliquetas (SigVALDADÓTTIR et al., 1997; FAUCHALD \& RousE, 1997), essas duas estruturas foram aqui consideradas homólogas. Spionidae e Paraonidae são os únicos grupos que possuem antena mediana.

35. Nervos palpais: 0 , ausente; 1 , presente.

Spionidae, Scalibregmatidae e Paraonidae apresentam nervos palpais no interior do prostôsmio, apesar de Scalibregmatidae não ter qualquer palpo exterior (FAUCHALD \& Rouse, 1997; Rouse \& PleiJel, 2001).

Foram obtidas 1440 árvores mais parcimoniosas com um comprimento de 70 passos, índice de consistência igual a 0,671 e índice de retenção 0,921 . Posteriormente, foi utilizado o consenso estrito para obtenção de um cladograma, contendo apenas os clados que apareceram em todas as 1440 árvores mais parcimoniosas (fig. 1). A seguir rodou-se novamente a matriz utilizando pesagem sucessiva e posteriormente consenso estrito (fig. 2).

A análise demonstrou que a família Questidae é monofilética, possuindo como sinapomorfias a posição dorso-lateral dos poros masculinos (caráter 23), par de grandes sacos espermáticos nos segmentos 12 e 13 (caráter 29) e par de vesículas seminais do segmento 14 conectado aos sacos espermáticos (caráter 30). A presença de brânquias dorsais pareadas não-ramificadas nos segmentos posteriores também pode ser interpretada como uma sinapomorfia, já que todas as espécies da família apresentam essa característica, sendo revertida secundariamente apenas em $Q$. trifurcata (fig. 2). As brânquias e a ausência de cirro anal no pigídio (caráter 28) de Q. trifurcata foram utilizadas para diagnosticar o gênero Novaquesta (Hobson, 1970; Somaschini \& Gravina, 1993; Delgado \& NuÑEz, 1994). Essas características são aqui interpretadas como autapomórficas de $Q$. trifurcata. Concordamos com a sinonimização de Novaquesta com Questa feita por GIERE \& ERséus (1998), uma vez que a manutenção de Novaquesta torna o gênero Questa parafilético.

Além dos relacionamentos internos demonstrados para Questidae, foi possível formular hipóteses de relacionamentos filogenéticos dos grupos Scolecida, Clitellata e de seus táxons internos. A análise filogenética demonstrou que os "Oligochaeta" são parafiléticos, bem como os oligoquetos aquáticos ou "Microdilli" e a família "Tubificidae". 


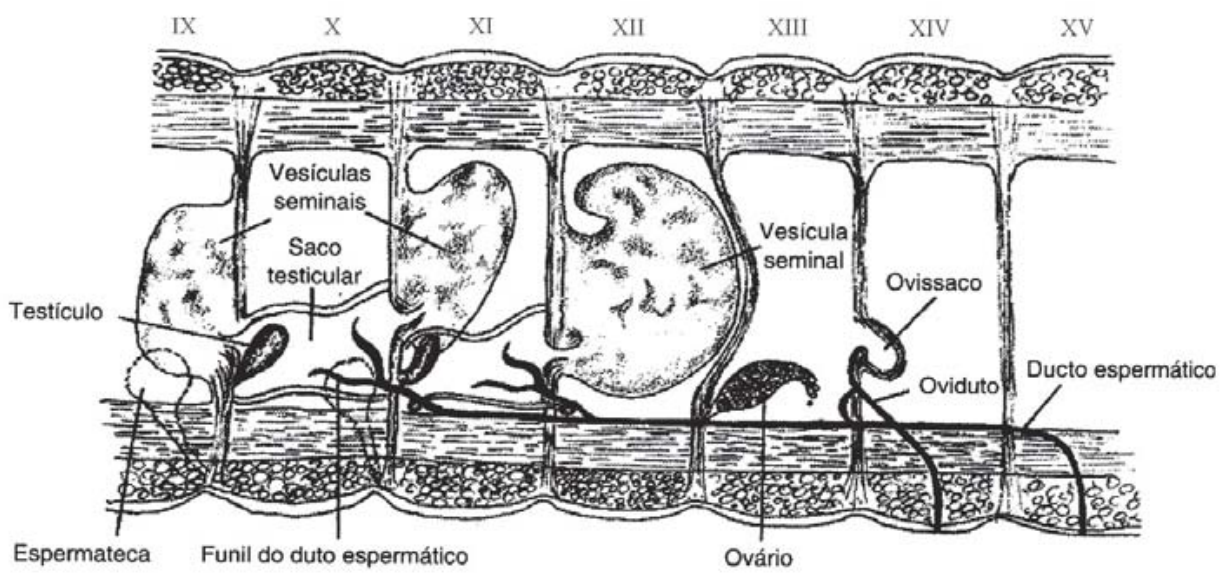

Fig. 9. Lumbricus terrestris Linnaeus, 1758, Megadrilii. Vista lateral interna dos tagmas que contém os órgãos reprodutivos (modificado de RUPPERT \& BARNES, 1996).

É interessante notar que tanto OMOdeo (1998) como Erseus $(1987,1990)$ e BrinKHruST (1991, 1994), utilizando a morfologia comparada, já haviam comentado, respectivamente, sobre a não monofilia dos Oligochaeta e dos "Microdilli" e da família "Tubificidae". A monofilia dos táxons Megadrilli e Hirudinae não parece apresentar contestações, pois trabalhos anteriores já haviam demonstrado a validade desses táxons (BRINKHURST, 1994; SidDAll \& BuRRESON, 1995). O táxon (Lumbriculidae (Achantobdellida (Branchiobdellida, Hirudinae))) também é considerado monofilético, fato já evidenciado anteriormente pela morfologia comparada (BRINKHURST, 1982, 1984b, 1994; BRINKHURST \& NEMEC, 1987).

Várias das conclusões obtidas por este trabalho estão baseadas na posição dos órgãos reprodutivos (caracteres 14-21) e na presença de um clitelo com funções reprodutivas (caráter 2). Esse último caráter é a sinapomorfia que une os Questidae aos Clitellata. A única diferença entre os dois grupos é a presença de clitelo apenas nas fêmeas dos questídeos, visto que este grupo apresenta os sexos separados.

Uma característica importante nesses organismos é a formação dos tagmas exclusivos para as estruturas reprodutivas. Em Spionida e Scolecida, as gônadas são dispersas, ou melhor, não possuem segmentos exclusivos para essas estruturas (já nas fases iniciais do desenvolvimento). Nos Questidae é encontrada, pela primeira vez, a organização em tagmas exclusivos para a reprodução, continuando inalterada em todos os demais integrantes dos Clitellata. As gônadas dos Questidae possuem dois pares (dois pares de ovários nas fêmeas e dois pares de testículos nos machos) (fig. 3), semelhantes ao número de órgãos reprodutivos encontrados na família mais basal dos Clitellata, os Haplotaxidae (fig. 4). A posição das gônadas nas duas famílias é muito semelhante. Nos Haplotaxidae, as gônadas masculinas encontram-se nos segmentos $10 \mathrm{e}$ 11 e as femininas nos 12 e 13. Nos questídeos, apenas a localização dos órgãos reprodutores masculinos difere em posição em relação aos de Haplotaxidae, estando nos segmentos 11 e 12 e os femininos nos 12 e 13. Ambos apresentam a condição dita plesiópora (fig. 5), ou seja, o testículo e o início do gonoducto estão no segmento posterior ao gonóporo (BRINKHURST, 1982). A partir daí, derivaram-se duas condições apomórficas, a opistópora 
e a prosópora (fig. 5). A condição opistópora ocorre quando o ducto espermático atravessa pelo menos um segmento inteiro do corpo, sendo este segmento localizado entre o segmento que contém o início do ducto e o segmento do testículo (BRINKHURST, 1982). A condição prosópora ocorre quando o testículo, o início do gonoducto e o gonóporo estão no mesmo segmento (BRINKHURST, 1982). A partir disso, inferimos a homologia primária (sensu De PinNa, 1991) entre os diferentes estados dos aparelhos reprodutores encontrados e obtivemos, na filogenia final, dois grupos distintos que diferenciaram da condição plesiomórfica (plesiópora) encontrada nos "Microdilli": os Megadrilli (opistópora) e os Lumbriculidae + Hirudinae (prosópora) (figs. 6-8). Juntamente com estas alterações na forma das estruturas reprodutoras, ocorreram alterações no número de gônadas nos diferentes táxons. Nos "Oligochaeta", a partir de uma condição simplesiomórfica (presença de dois pares de gônadas femininas), surgiram duas condições sinapomórficas independentes: a perda do primeiro par de ovários nos Megadrilli (fig. 9) e a perda do segundo par nos "Microdilli" e Hirudinea, caracteres que suportam a formação de dois táxons distintos, ou seja, ((Megadrilli), ("Microdilli", (Lumbriculidae, Hirudinae)).

A hipótese de evolução dos animais com clitelo considerada nesse trabalho é similar àquela proposta por BRINKHURST (1984a, 1984b), na qual o autor utilizou o grau de desenvolvimento do átrio e a glândula prostática como caracteres delineadores.

Com relação aos demais poliquetas analisados, encontramos grupos monofiléticos menores, contudo, muitos formados a partir de homoplasias (fig. 2). Para a construção da matriz de dados de Scolecida foram utilizados, em grande parte, os caracteres mencionados por Rouse \& FAUCHALD (1997). Em Scolecida obtivemos a seguinte relação filogenética: (Paraonidae (Scalibregmatidae ((Opheliidae, Maldanidae) (Capitellidae, Arenicolidae) Orbiniidae, Cossuridae))). A hipótese ora proposta é que Scolecida, no sentido proposto por RouSE \& FAUCHALD (1997), é parafilético, uma vez que esse grupo exclui os Clitellata.

Agradecimentos. A Martin L. Christoffersen (UFPB), por ser autor intelectual do problema, fornecer a bibliografia inicial e laboratório para o desenvolvimento de parte desse projeto. A Antônio C. Marques (USP), Pedro Martinez (UFPR), Cecília Amaral (UNICAMP), Cinthya Santos (UFPR), Waltécio de Oliveira Almeida (UFPB), Paulo Lana (UFPR) e a dois revisores anônimos pelos valorosos comentários efetuados; à FAPESP pelo apoio financeiro (FAPESP: 97/06495-7).

\section{REFERÊNCIAS BIBLIOGRÁFICAS}

Brinkhurst, R. O. 1982. Evolution in the Annelida. Can. J. Zool., Toronto, 60:1043-1059. 1984a. Comments on the evolution of the Annelida. Hydrobiologia, Dordrecht, 109:189-191.

-. 1984b. The position of the Haplotaxidae in the evolution of Oligochaeta annelids. Hydrobiologia, Dordrecht, 115:25-36.

1991. A phylogenetic analysis of the Tubificinae (Oligochaeta: Tubificidae). Can. J. Zool., Toronto, 69:392-397.

1992. Evolutionary relationships within the Clitellata. Soil Biol. Biochem., Toronto, 12:12011205.

1994. Evolutionary relationships within the Clitellata: an update. Megadrilogica, Rindsay, 5 (10):109-112.

Brinkhurst, R. O. \& Jamieson, B. G. M. 1971. Aquatic Oligochaeta of the world. Edinburgh, Oliver \& Boyd. 860p.

Brinkhurst, R. O. \& NemEc, A. F. L. 1987. A comparison of phenetic and phylogenetic methods applied to the systematic of Oligochaeta. Hydrobiologia, Dordrecht, 155:65-74.

Delgado, J. D. \& Nuñez, J. 1994. Presencia de Questa caudicirra Hartman, 1966 (Polychaeta, Questidae) en las Islas Canarias. Misc. Zool., Barcelona, 17:272-277. 
De Pinna, M. C. C. 1991. Concepts and test of homology in the cladistic paradigm. Cladistics, San Diego, 7:367-394.

ERséus, C. 1987. Phylogenetic analysis of the aquatic Oligochaeta under the principle of parsimony. Hydrobiologia, Dordrecht, 155:75-89.

1990. Cladistic analysis of the subfamilies within the Tubifidae (Oligochaeta). Zool. Scr., Götemborg, 19:57-63.

FARRIS, J. S. 1969. A successive approximations approach to character weighting. Syst. Zool., Storrs, 18:374-385.

1989. The retention index and the rescaled consistency index. Cladistics, San Diego, 5:417-419.

Fauchald, K. \& Rouse, G. W. 1997. Polychaeta systematics: Past and present. Zool. Scr., Götemborg, 26:71-138.

Giere, O. W. \& Erséus, C. 1998. A systematic account of the Questidae (Annelida, Polychaeta), with description of the new taxa. Zool. Scr., Götemborg, 27:345-360.

Giere, O. W. \& Rise, N. W. 1981. Questidae: polychaetes with oligochaetoid morphology and development. Zool. Scr., Götemborg, 10:95-105.

Glasby, C. J. 2000. Questidae. In: Glasby, C. J.; Hutchings, P. A. et al. Fauna of Australia. Annelida through Tunicata. Sydney, Australian Biological Resources Study. v.4, 465p.

Hartman, O. 1966. Quantitative survey of the benthos of San Pedro basin, southern California. Part 2. Final results and conclusions. Allan Hancock Pacific Exped., Los Angeles, 19:187-456.

Hobson, K. D. 1970. Novaquesta trifurcata. A new genus and species of family Questidae (Annelida: Polychaeta). Proc. Biol. Soc. Wash., Washington, 83:191-194.

JAmieson, B. G. M. 1983a. Spermiogenesis in the olygochaetoid polychaete Questa (Annelida, Questidae). Zool. Scr., Götemborg, 12:179-186.

1983b. The ultrastructure of the spermatozoon of the oligochaetoid polychaete Questa sp. (Questidae, Annelida) and its phylogenetic significance. J. Ultrastruct. Res., Orlando, 84:238-251.

Jamieson, B. G. M. \& WeBB, R. I. 1984. The morphology, spermatozoal ultrastructure and phylogenetic affinities of new species of questid (Polychaeta: Annelida). In: Polychaeta Conference, $1^{\circ}$, Proceedings... Linnean Society of New South Wales, New South Wales, p.21-34.

Nixon, K. C. \& Carpenter J. M. 1993. On outgroup. Cladistics, San Diego, 9:413-426.

Omodeo, P. 1998. History of Clitellata. Ital. J. Zool., Firenze, 65:51-73.

Pettibone, M. H. 1982. Annelida. In: Parker, S. P. Synopsis and classification of living organism. New York, McGraw-Hill. p.27, 28.

PuRschKe, G. 1997. Ultrastructure of nuchal organs in polychaetes (Annelida); new results and review. Acta Zool., Stockholm, 78:123-143.

. 1999. Terrestrial polychaetes- models for the evolution of Clitellata (Annelida)? Hydrobiologia, Dordrecht, 406:87-99.

Rouse, G. W. \& Fauchald, K. 1997. Cladistics and polychaetes. Zool. Scr., Götemborg, 26:139-204.

Rouse, G. W. \& Pleijel, P. 2001. Polychaetes. London, Oxford. 354p.

Ruppert, E. E. \& Barnes, R. D. 1996. Zoologia dos invertebrados. São Paulo, Roca. 1029p.

Siddall, M. E, \& Burreson, E. M. 1995. Phylogenetic of the Euhirudinea: Independent evolution of blood feeding by leeches? Can. J. Zool., Toronto, 73:1054-1064.

SigvaldadóttiR, E., Mackei, A. S. Y. \& PleiJel, F. 1997. Generic interrelationships within the Spionidae: Annelida: Polycheta). Zool. J. Linn. Soc., Londres, 119:473-500.

Sokal, R. R. \& Rohlf, F. J. 1981. Taxonomic congruence in the Lepopodomorpha reexamined. Syst. Zool., Storrs, 30:309-325.

Somaschini, A. \& Gravina, M. F. 1993. First report of Questidae (Annelida, Polychaeta) in the Mediterranean sea. Vie et Milieu, Paris, 43(1):59-61.

Westheide, W. 1981. Interstitial fauna of Galapagos XXVI. Questidae, Cirratulidae, Acrocirridae, Ctenodrilidae (Polychaeta). Mikrof. Meeresb., Stuttgart, 82:59-79.

Wilkinson, M. 1992. Ordered versus unordered characters. Cladistics, San Diego, 8:375-385.

- 1995. A comparison of two methods of character construction. Cladistics, San Diego, 11:297308.

Recebido em 25.03.2002; aceito em 09.12.2002. 